Part of Journal of Research of the National Bureau of Standards, Volume 31, August 1943

\title{
TENSILE AND OTHER PROPERTIES OF CONCRETES MADE WITH VARIOUS TYPES OF CEMENTS
}

\author{
By Louis Schuman and John Tucker, Jr.
}

\begin{abstract}
An improved tensile-test method for concrete has been developed. Cylindrical specimens, 4 by 16 inches, are used, and loads are applied at both ends through threaded rods embedded in a rich mortar. Compressive- and tensile-strength and stress-strain determinations have been made for concretes made with various types of cements, including cements with aerating agents, and with various aggregates. At early ages, strengths for moderate-heat cements were but slightly lower than for normal cements, whereas those for high-early-strength cements were considerably higher. Tensile strengths usually attained maximum values between 7 days and 3 months. Tensile strengths depended on compressive strengths, type of cement, type of aggregate, and on sand-gravel ratios.
\end{abstract}

\section{CONTENTS}

Page

I. Introduction

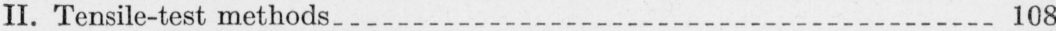

1. Previous methods

2. Improved method $\ldots \ldots \ldots \ldots$

III. Materials and tests

1. Cements _ _.

2. Mixes and tests

IV. Preparation of test specimens

1. Compressive specimens

2. Tensile specimens

V. Test methods

1. Compressive and tensile tests

2. Stress-strain determinations $\ldots$

VI. Test results and discussion _........... 112

1. Concrete made with various cements_............ 112

(a) General

(b) Variation in strength for cements within a type

(c) Variation in strength with type of cement_........ 116

(d) Effect of age of concrete on strength $\ldots \ldots \ldots \ldots . . . . .117$

(e) Effect on strength of cement content of concrete ..... 118

(f) Relations between compressive and tensile strengths _ 119

(g) Stress-strain relations _....... 119

2. Effect of sand-gravel ratio and of various coarse aggregates on tensile strength

(a) Sand-gravel ratio

(b) Coarse aggregates........ 121

VII. Summary

VIII. References 


\section{INTRODUCTION}

The tensile strength of concrete is an important factor in its resistance to fracture by bending, shrinkage, freezing and thawing, or differential expansion. Although a number of investigations on both tensile and flexural properties of concretes have been reported, no comparative data on the effect of the newer types of cement are available. The present investigation was undertaken to develop a more satisfactory tensile-test method for concrete than has been used in the past, and particularly to secure data on the compressive, tensile, and stress-strain properties of concretes made with various types of cement, including cements with aerating agents. In addition, the effect of varying the sand-gravel ratio or the type of coarse aggregate on the tensile strength of concrete was studied.

\section{TENSILE-TEST METHODS}

\section{PREVIOUS METHODS}

Unlike the compressive test, which has been standardized [1], ${ }^{1}$ tensile tests of concrete have been made by a number of different methods [2 to 9]. The dimensions of the specimens have ranged from 12 to $39 \mathrm{in}$. in length, and from 3 to 6 in. square, or in diameter. The shapes of the specimens have also varied, depending on the method of applying the load. Some investigators $[4,7,8,9]$ used specimens with enlarged ends to which grips were applied. Others $[5,11]$ cast cylindrical specimens and used grips clamped or wedged to the ends of the test pieces. Besides the use of unduly large specimens, some of these methods are open to objection because of the way the specimens break. Specimens with enlarged ends often break at the junction of the enlarged and reduced portions. Cylindrical specimens, on the other hand, may break near or within the grips [5].

\section{IMPROVED METHOD}

The type of specimen and method of loading developed in the present investigation are believed to overcome these objections. In principle, the method is similar to that used in the tests of Talbot and of Withey [2,3].

The new feature of the improved method of determining tensile strength is the application of the load to the concrete under test through threaded rods embedded in a rich mortar molded on the ends of the cylindrical test specimens (fig. 1). The preliminary tests with this method showed that the specimens always broke well within the concrete under test, and that a bond stress of $700 \mathrm{lb} / \mathrm{in}^{2}{ }^{2}$ of embedded rod area could be safely used. Thus, for the rods in figure 1, giving about 12 in. $^{2}$ of bond area, no failure in bond or at the ends of the rods will occur for loads up to $8,000 \mathrm{lb}$., equivalent to a tensile stress in the concrete of $600 \mathrm{lb} / \mathrm{in} .{ }^{2}$.

A few tests were made to compare the new method with certain of the older methods. With a $1: 4.5$ concrete, six specimens were molded and tested by the new method, and six by each of the following methods:

\footnotetext{
1 Figures in brackets indicate the literature references at the end of this paper.
} 

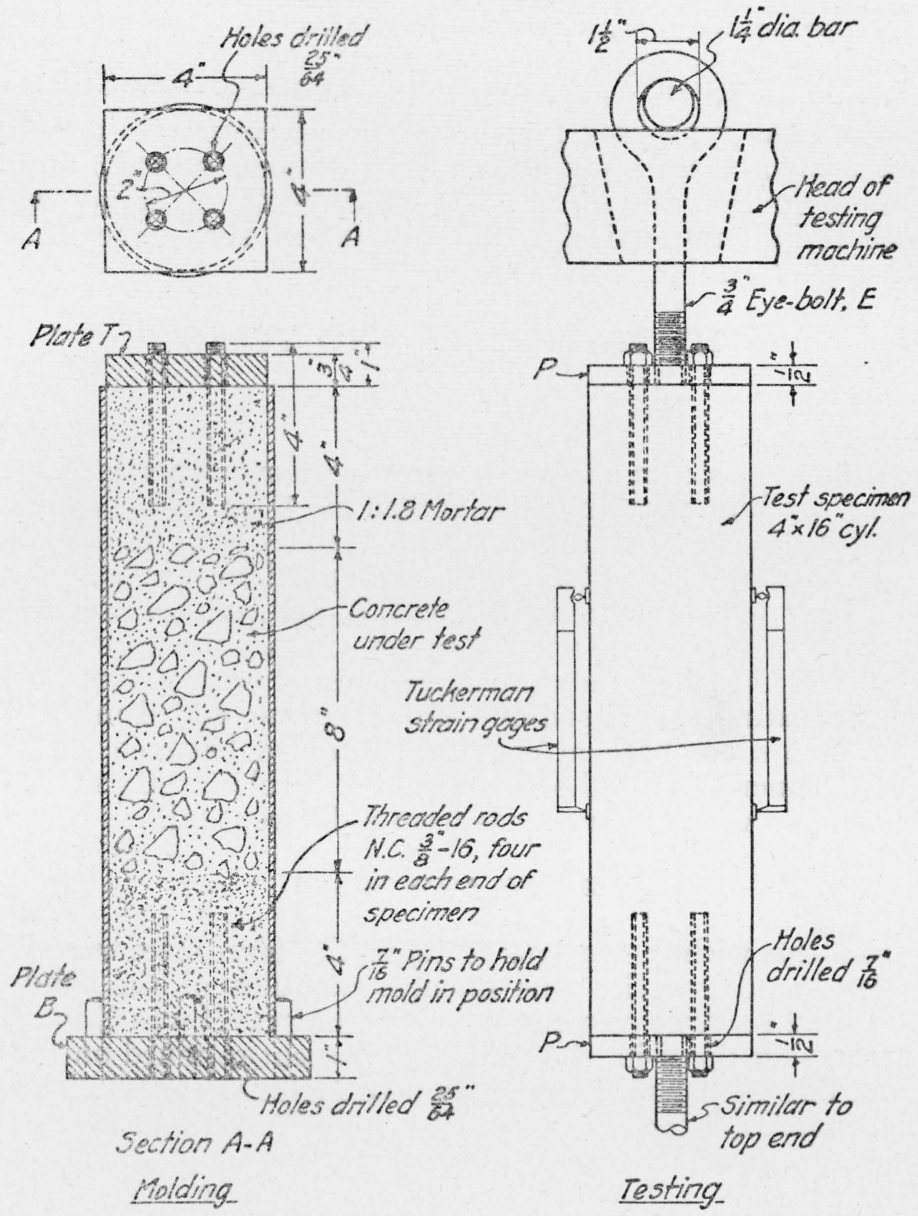

Figure 1.-Method of molding and of testing tensile specimens.

1. Specimens $4 \frac{1}{2}$ in. in diameter, with splayed ends; space between grips and specimens filled with a plastic material. Method similar to Johnson's [4].

2. Specimens 3 in. in diameter, plain rods of unequal lengths embedded directly in the ends of the concrete; load applied through plates attached to the rods at each end of the specimen. Method similar to Davis's [6].

By method 1, the values of tensile strength obtained ranged from 350 to $420 \mathrm{lb} / \mathrm{in}^{2}$, with an average of $380 \mathrm{lb} / \mathrm{in}^{2}$.

By method 2, the values ranged from 245 to $380 \mathrm{lb} / \mathrm{in}^{2}{ }^{2}$, with an average of $320 \mathrm{lb} / \mathrm{in}^{2}$.

The values obtained by the new method ranged from 400 to 420 $\mathrm{lb} / \mathrm{in}^{2}$, with an average of $430 \mathrm{lb} / \mathrm{in}^{2}$.

The higher value obtained by the new method is believed to indicate a more uniform stress distribution across the section of failure than with the other methods. 
A 1:3 mortar made with standard Ottawa sand, as used in cement testing (Federal Specification SS-C-158a), was also tested. Mortar from the same batch was molded into standard briquets and into cylinders of the type used for the new concrete tensile-test method. At 7 days the average strength for three briquets was $295 \mathrm{lb} / \mathrm{in} .^{2}$, and for three cylinders, $360 \mathrm{lb} / \mathrm{in}^{2}{ }^{2}$. At 28 days the averages were 415 and $565 \mathrm{lb} / \mathrm{in}^{2}{ }^{2}$, respectively. The cylinders thus gave 22 and 36 percent higher strengths than the briquets.

\section{MATERIALS AND TESTS}

\section{CEMENTS}

The cements used in the tests were five normal portland cements, including a white portland, designated $\mathrm{N}-1$ to $\mathrm{N}-5$, table 1 ; four moderate-heat portland cements, $\mathrm{M}-1$ to $\mathrm{M}-4$; three high-earlystrength portland cements, S1-S3; two portland-pozzolan cements, $\mathrm{P}-1$ and $\mathrm{P}-2$; one high-alumina cement, $\mathrm{L}$; and eight portland cements with rosin, tallow, or vinsol resin as aerating agents ground in at the mill, table 2, C to J. One cement was tested both without and with a soluble aerating agent, sodium lauryl sulfate, added in the laboratory, so that a comparison could be obtained between aerated and unaerated concretes for the same cement, table 2, A and B. Each of the cements within a type was from a different mill.

\section{MIXES AND TESTS}

For the tests with the various cements, the aggregates were Potomac River sand and gravel. The fineness modulus of the sand varied from 2.6 to 2.8. The maximum size of the gravel was $3 / 4$ in., with 34 percent passing the $3 / 8$-in. sieve and 100 percent retained on the No. 4 .

For all but the aerated concretes, two mixes were tested with each of the cements, a $1: 2.4: 3.6$ by weight $(\operatorname{mix} B, 1: 6)$ and a $1: 1.75: 2.75$ by weight (mix $H, 1: 4.5$ ). Only mix $B$ was used in the tests on aerated concretes. Compressive and tensile specimens, in triplicate, were made for test at 3,7 , and 28 days, 3 months, and 1 year, except that for the high-early-strength and high-alumina cements tests at 1 day were added and the 3-month compressive tests omitted. In addition to the compressive and tensile tests, stress-strain relations were determined on the concretes made with the cements containing aerating agents.

To study the effect of varying the aggregate proportions, a series of tensile tests was made in which the sand-gravel ratio in a 1:6 mix with the Potomac aggregates was varied. These tests were made with each of three different portland cements. The proportions varied from $1: 1.9: 4.1$ to $1: 3.4: 2.6$, and the water content was varied correspondingly to maintain approximately equal flow values. Tensile strengths at 3 and 28 days were determined.

Another series of tests was made to study the effect of a few different coarse aggregates. For this series, a single cement with Potomac sand was used. The coarse aggregates were Potomac gravel, a New York limestone, an Ohio slag, a Pennsylvania traprock, and a New York granite. Two mixes were tested, except for the granite and trap-rock, for which there was only sufficient material 
for one mix. For the Potomac gravel, the mixes were the same as for the tests of the various cements. For the other coarse aggregates, the cement-sand ratios were the same as for the Potomac, but the proportions of coarse aggregate were varied with the bulk specific gravities so as to give approximately equal yields of concrete. The water contents were varied to give equal flow values, except in one case where the Potomac gravel was tested in a mix with the same $C / W$ ratio as was used for the other aggregates. Tests were made on the $1: 6$ mixes at 7 and 28 days, and on the 1:4.5 mixes at 3 and 28 days (table 4).

In all of the above tests, a set of three specimens was tested at each test age. The cements used in the series of tests with various aggregates and sand-gravel ratios were of different brands or lots from those mentioned in section III-1.

\section{PREPARATION OF TEST SPECIMENS}

\section{COMPRESSIVE SPECIMENS}

All mixing and molding of the test specimens was done in a laboratory maintained at $70^{\circ} \pm 3^{\circ} \mathrm{F}$. The 6 by $12 \mathrm{in}$. compression cylinders were prepared by ASTM method C39-39 [1]. The mixing time was generally 2 minutes. It was found, however, that the 2 minutes of hand mixing was not sufficient to incorporate substantial amounts of air where the cements contained aerating agents. Hence these concretes were mixed for 3 minutes.

Flow values were determined by ASTM method C124-39, [1, P. 337] except that the mold was filled without rodding, and the table dropped 15 times through a distance of $1 / 8 \mathrm{in}$. The cement-water ratio was generally 1.66 for the $1.6 \mathrm{mix}$ and 2.05 for the $1: 4.5 \mathrm{mix}$ (6.8 and 5.5 gal/bag, respectively) but was varied somewhat to maintain approximately equal flows.

The tops of the compression cylinders were capped with neatcement paste, not less than 4 hours after molding them. For the slower-setting cements, capping the cylinders in less than 4 hours after molding, results in distortion of the caps.

The cylinders were left in the laboratory for 24 hours, then removed from the molds and stored in the damp room at $70^{\circ} \pm 3^{\circ} \mathrm{F}$ until tested.

\section{TENSILE SPECIMENS}

The method of molding the tensile-test specimens can be seen in figure 1 . The $3 / 8$ - by 4 -in. threaded rods were held in position by the holes in the bottom plate, $B$, and the mold was held in place by the pins in the plate. The mortar for the ends of the tensile specimen consisted of the test cement and sand, $1: 1.8$ by weight, with a $C / W$ (cement-water) ratio of 2.4 to 2.6. Sufficient mortar for a set of three tensile specimens was mixed in a pan, by hand. The lower ends of three specimens were then molded by rodding the mortar, in two layers, each about 2 in. in depth, so as to extend about 1 in. above the ends of the threaded rods, figure 1 . Concrete, sufficient for 3 specimens, was then mixed and placed in the mold in two 4-in. layers, each rodded with 20 strokes of the tamping rod. The remaining mortar was then placed and rodded into the top of each mold. 


\section{Journal of Research of the National Bureau of Standards}

The plates, $T$, were then placed in position over the tops of the molds and the threaded rods inserted through the holes, with some turning and vertical motion to secure good bond to the mortar. The rods extended $1 \mathrm{in}$. beyond each end of the specimen to provide for the plates used in testing, figure 1, and had a length of 3 in. embedded in the mortar. The space between the ends of the rods and the concrete contained a mixture of mortar and concrete.

The threaded rods were lightly greased before molding into the tensile specimens, and could be readily removed by splitting the cylinders. The same rods were then used in other specimens.

The tensile specimens were stored and cured under the same conditions as the compressive specimens.

\section{TEST METHODS}

\section{COMPRESSIVE AND TENSILE TESTS}

The compressive tests were made in a 300,000-lb. hydraulic testing machine at a rate of loading of $1,000 \mathrm{lb}$./in. ${ }^{2}$ a minute. The tensile tests were made in a 100,000-lb. beam-and-poise, screw-driven testing machine, operating at an idle head speed of 0.05 in. per minute. Onehalf-inch plates, $P$, figure 1 , were attached to the specimen, and eyebolts, $E$, attached to the plates. The loads were applied through bars passing through the eye of each bolt and resting against the testing-machine heads.

\section{STRESS-STRAIN DETERMINATIONS}

Strains were measured with two Tuckerman optical strain gages [10], extended to a 6-in. gage length. The gages were seated on thin brass plates attached with plaster of paris to the specimen, and were diametrically opposite each other. An intitial stress of 50 or $100 \mathrm{lb} /$ in. ${ }^{2}$ for the compressive specimens, or of $10 \mathrm{lb} / \mathrm{in} .^{2}$ for the tensile specimens, was applied for the first readings. Rates of loading were the same as for the strength tests, but the loads were maintained as nearly constant as possible while the gages were being read. Strains were measured to less than one-millionth.

However, because of the difficulty of maintaining constant loads with the hydraulic machine, any load increment may be in error by as much as $400 \mathrm{lb}$, equivalent to $14 \mathrm{lb} /$ in. $^{2}$ on a 6 - by 12 -in. cylinder, corresponding to a strain error of $4 \times 10^{-6}$ for a modulus of $3.5 \times 10^{6} \mathrm{lb} /$ in. $^{2}$ This error is less than 2 percent of the strain at a stress of 1,000 $\mathrm{lb} /$ in $^{2}$, and correspondingly lower at higher stresses.

\section{TEST RESULTS AND DISCUSSION}

\section{CONCRETES MADE WITH VARIOUS CEMENTS}

(a) GENERAL

The test results for two concrete mixes with the various cements, and for the aerated concretes, are given in tables 1 and 2 . For any one specimen of a set of three, the deviation from the average was usually less than 10 percent, both for the tensile and compressive specimens. Practically none of the tensile fractures occurred at a distance of less than 2 in. from the ends of the threaded rods, and about 80 percent of the fractures were within 2 in. of the middle of the specimen. 
$T_{A B L E}$ 1.-Compressive and tensile strengths of concretes made with various types of cement

[Each strength value is the average for 3 test specimens]

\begin{tabular}{|c|c|c|c|c|c|c|c|c|c|c|c|c|c|c|c|}
\hline \multirow{2}{*}{ Type of cement } & \multirow{2}{*}{$\begin{array}{l}\text { Identifi- } \\
\text { cation } \\
\text { number }\end{array}$} & \multirow{2}{*}{$\begin{array}{l}C / W^{2} \text { by } \\
\text { weight }\end{array}$} & \multirow{2}{*}{ Flow } & \multicolumn{6}{|c|}{ Compressive strength (lb/in..$\left.^{2}\right)$ at- } & \multicolumn{6}{|c|}{ Tensile strength (lb/in..$\left.^{2}\right)$ at- } \\
\hline & & & & 1 day & 3 day & 7 day & 28 day & $3 \mathrm{mo}$. & $1 \mathrm{yr}$. & 1 day & 3 day & 7 day & 28 day & $3 \mathrm{mo}$. & $1 \mathrm{yr}$. \\
\hline \multicolumn{16}{|c|}{ MIX $B .1: 6$ BY WEIGHT } \\
\hline $\begin{array}{l}\text { Normal } \\
\text { Do } \\
\text { Do } \\
\text { Do } \\
\text { Normal (white) }\end{array}$ & \multirow[t]{2}{*}{$\begin{array}{l}\mathrm{N}-1 \\
\mathrm{~N}-2 \\
\mathrm{~N}-3 \\
\mathrm{~N}-4 \\
\mathrm{~N}-5\end{array}$} & \multirow[t]{2}{*}{$\begin{array}{l}1.66 \\
1.66 \\
1.66 \\
1.66 \\
1.66\end{array}$} & \multirow[t]{2}{*}{$\begin{array}{l}\% \\
48 \\
53 \\
48 \\
45 \\
55\end{array}$} & 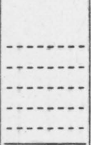 & $\begin{array}{l}1,490 \\
1,420 \\
1,520 \\
1,490 \\
1,560\end{array}$ & $\begin{array}{l}2,260 \\
2,260 \\
2,730 \\
2,820 \\
2,660\end{array}$ & $\begin{array}{l}4,440 \\
4,300 \\
4,800 \\
5,030 \\
4,560\end{array}$ & $\begin{array}{l}5,030 \\
5,440 \\
5,420 \\
5,900 \\
5,040\end{array}$ & $\begin{array}{l}5,540 \\
5,960 \\
5,800 \\
6,160 \\
5,960\end{array}$ & \multicolumn{2}{|r|}{$\begin{array}{l}170 \\
170 \\
170 \\
200 \\
195\end{array}$} & $\begin{array}{l}240 \\
230 \\
255 \\
285 \\
290\end{array}$ & $\begin{array}{l}355 \\
415 \\
395 \\
440 \\
425 \\
\end{array}$ & $\begin{array}{l}360 \\
430 \\
365 \\
425 \\
385\end{array}$ & $\begin{array}{l}355 \\
450 \\
370 \\
400 \\
430\end{array}$ \\
\hline A verage... & & & & & 1,490 & 2,550 & 4,630 & 5,370 & 5,880 & 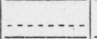 & 180 & 260 & 405 & 395 & 400 \\
\hline 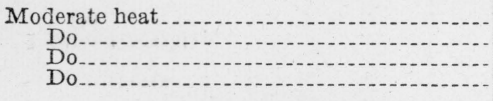 & \multirow[t]{2}{*}{$\begin{array}{l}M-1 \\
M-2 \\
M-3 \\
M-4\end{array}$} & \multirow[t]{2}{*}{$\begin{array}{l}1.66 \\
1.66 \\
1.66 \\
1.66\end{array}$} & \multirow[t]{2}{*}{$\begin{array}{l}57 \\
46 \\
50 \\
50\end{array}$} & 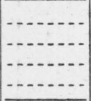 & $\begin{array}{l}1,030 \\
1,040 \\
1,440 \\
1,380\end{array}$ & $\begin{array}{l}1,760 \\
2,170 \\
2,510 \\
2,350 \\
\end{array}$ & $\begin{array}{l}3,550 \\
4,560 \\
4,420 \\
4,480 \\
\end{array}$ & $\begin{array}{l}5,560 \\
5,250 \\
5,330 \\
5,460\end{array}$ & $\begin{array}{l}6,530 \\
6,480 \\
6,340 \\
5,800\end{array}$ & 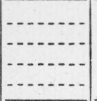 & $\begin{array}{l}135 \\
150 \\
195 \\
190 \\
\end{array}$ & $\begin{array}{l}200 \\
260 \\
285 \\
275\end{array}$ & $\begin{array}{l}355 \\
395 \\
385 \\
430\end{array}$ & $\begin{array}{l}435 \\
375 \\
405 \\
405 \\
\end{array}$ & $\begin{array}{l}470 \\
435 \\
445 \\
405 \\
\end{array}$ \\
\hline A verage.... & & & & - & 1,220 & 2,200 & 4,250 & 5,400 & 6,290 & 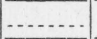 & 170 & 255 & 390 & 405 & 440 \\
\hline $\begin{array}{l}\text { Portland-pozzolan } \\
\text { Do }\end{array}$ & \multirow[t]{2}{*}{$\begin{array}{l}\mathrm{P}-1 \\
\mathrm{P}-2\end{array}$} & \multirow[t]{2}{*}{$\begin{array}{l}1.66 \\
1.55\end{array}$} & \multirow[t]{2}{*}{$\begin{array}{l}47 \\
52\end{array}$} & 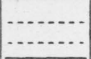 & $\begin{array}{r}800 \\
1,140\end{array}$ & $\begin{array}{l}2,060 \\
1,910\end{array}$ & $\begin{array}{l}4,670 \\
3,000 \\
\end{array}$ & $\begin{array}{l}5,530 \\
3,900 \\
\end{array}$ & $\begin{array}{l}5,860 \\
4,270\end{array}$ & - n & $\begin{array}{l}135 \\
160\end{array}$ & $\begin{array}{l}230 \\
235\end{array}$ & $\begin{array}{l}415 \\
315\end{array}$ & $\begin{array}{l}370 \\
310\end{array}$ & $\begin{array}{l}410 \\
355 \\
\end{array}$ \\
\hline A verage.... & & & & n........ & 970 & 1,980 & 3,830 & 4,710 & 5,070 & 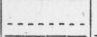 & 150 & 230 & 365 & 340 & 380 \\
\hline $\begin{array}{l}\text { High-early strength } \\
\text { Do } \\
\text { Do }\end{array}$ & \multirow[t]{2}{*}{$\begin{array}{l}\text { S-1 } \\
\text { S-2 } \\
\text { S-3 }\end{array}$} & \multirow[t]{2}{*}{$\begin{array}{l}\text { 1. } 66 \\
\text { 1. } 59 \\
1.66\end{array}$} & \multirow[t]{2}{*}{$\begin{array}{l}49 \\
54 \\
44\end{array}$} & $\begin{array}{l}1,020 \\
1,150 \\
1,020 \\
\end{array}$ & $\begin{array}{l}2,580 \\
2,730 \\
3,310 \\
\end{array}$ & $\begin{array}{l}3,690 \\
3,980 \\
4,280 \\
\end{array}$ & $\begin{array}{l}5,420 \\
5,500 \\
6,150 \\
\end{array}$ & 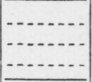 & $\begin{array}{l}5,690 \\
5,280 \\
5,970\end{array}$ & $\begin{array}{l}150 \\
160 \\
155 \\
\end{array}$ & $\begin{array}{l}300 \\
300 \\
365 \\
\end{array}$ & $\begin{array}{l}430 \\
435 \\
445\end{array}$ & $\begin{array}{l}505 \\
460 \\
480\end{array}$ & $\begin{array}{l}410 \\
365 \\
420 \\
\end{array}$ & $\begin{array}{l}435 \\
365 \\
390 \\
\end{array}$ \\
\hline A verage... & & & & 1,060 & 2,870 & 3,980 & 5,690 & -......... & 5,650 & 155 & 320 & 435 & 480 & 400 & 395 \\
\hline High alumina_.--. & $\mathbf{L}$ & 1.66 & 58 & 4,030 & 4,270 & 4,980 & 5,510 & 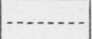 & 4,130 & 295 & 330 & 290 & 275 & -... & 220 \\
\hline
\end{tabular}

See footnotes at end of table. 
TABLE 1.-Compressive and tensile strengths of concretes made with various types of cement-Continued

\begin{tabular}{|c|c|c|c|c|c|c|c|c|c|c|c|c|c|c|c|}
\hline \multirow{2}{*}{ Type of cement } & \multirow{2}{*}{$\begin{array}{l}\text { Identifi- } \\
\text { cation } 1 \\
\text { number }\end{array}$} & \multirow{2}{*}{$\begin{array}{c}C / W^{2} \text { by } \\
\text { weight }\end{array}$} & \multirow{2}{*}{ Flow } & \multicolumn{6}{|c|}{ Compressive strength (lb/in..$\left.^{2}\right)$ at- } & \multicolumn{6}{|c|}{ Tensile strength $\left(\mathrm{lb} / \mathrm{in}^{2}{ }^{2}\right)$ at- } \\
\hline & & & & 1 day & 3 day & 7 day & 28 day & $3 \mathrm{mo}$. & $1 \mathrm{yr}$. & 1 day & 3 day & 7 day & 28 day & $3 \mathrm{mo}$. & $1 \mathrm{yr}$. \\
\hline \multicolumn{16}{|c|}{ MIX $H .1: 4.5$ BY WEIGHT } \\
\hline $\begin{array}{l}\text { Normal } \\
\text { Do- } \\
\text { Do- } \\
\text { Do } \\
\text { Normal (white) }\end{array}$ & \multirow[t]{2}{*}{$\begin{array}{l}\mathrm{N}-1 \\
\mathrm{~N}-2 \\
\mathrm{~N}-3 \\
\mathrm{~N}-4 \\
\mathrm{~N}-5\end{array}$} & \multirow[t]{2}{*}{$\begin{array}{l}2.05 \\
2.05 \\
2.05 \\
2.05 \\
2.05\end{array}$} & \multirow[t]{2}{*}{$\begin{array}{l}\% \\
49 \\
52 \\
53 \\
52 \\
59\end{array}$} & 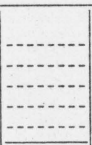 & $\begin{array}{r}2,210 \\
2,030 \\
2,380 \\
2,280 \\
2,390 \\
\end{array}$ & $\begin{array}{l}3,360 \\
3,230 \\
3,990 \\
4,070 \\
3,450 \\
\end{array}$ & $\begin{array}{l}5,270 \\
5,360 \\
5,900 \\
6,620 \\
5,290\end{array}$ & $\begin{array}{l}6,010 \\
6,790 \\
6,610 \\
7,050 \\
6,130 \\
\end{array}$ & $\begin{array}{l}6,650 \\
7,910 \\
7,700 \\
8,030 \\
7,480\end{array}$ & \multicolumn{2}{|r|}{$\begin{array}{l}265 \\
250 \\
265 \\
300 \\
250\end{array}$} & $\begin{array}{l}390 \\
360 \\
435 \\
410 \\
350\end{array}$ & $\begin{array}{l}465 \\
530 \\
470 \\
515 \\
460\end{array}$ & $\begin{array}{l}425 \\
530 \\
505 \\
500 \\
465\end{array}$ & $\begin{array}{l}455 \\
525 \\
490 \\
485 \\
515\end{array}$ \\
\hline A verage & & & & 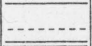 & 2,270 & 3,630 & 5,690 & 6,520 & 7,550 & $\ldots$ & 265 & 390 & 490 & 485 & 495 \\
\hline $\begin{array}{l}\text { Moderate heat_. } \\
\text { Do } \\
\text { Do-a } \\
\text { Do }\end{array}$ & \multirow[t]{2}{*}{$\begin{array}{l}M-1 \\
M-2 \\
M-3 \\
M-4\end{array}$} & \multirow[t]{2}{*}{$\begin{array}{l}2.05 \\
2.05 \\
2.05 \\
2.05\end{array}$} & \multirow[t]{2}{*}{$\begin{array}{l}61 \\
47 \\
42 \\
40\end{array}$} & 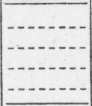 & $\begin{array}{l}1,670 \\
1,760 \\
2,100 \\
2,110\end{array}$ & $\begin{array}{l}2,780 \\
3,250 \\
3,620 \\
3,390 \\
\end{array}$ & $\begin{array}{l}5,020 \\
5,830 \\
5,230 \\
5,860\end{array}$ & $\begin{array}{l}6,880 \\
6,750 \\
6,580 \\
6,320\end{array}$ & $\begin{array}{l}8,400 \\
7,690 \\
7,820 \\
7,490 \\
\end{array}$ & \begin{tabular}{|c|} 
\\
-1 \\
\end{tabular} & $\begin{array}{l}230 \\
235 \\
285 \\
300 \\
\end{array}$ & $\begin{array}{l}310 \\
400 \\
440 \\
425 \\
\end{array}$ & $\begin{array}{l}520 \\
490 \\
480 \\
515 \\
\end{array}$ & $\begin{array}{l}535 \\
485 \\
465 \\
475 \\
\end{array}$ & $\begin{array}{l}595 \\
520 \\
545 \\
460 \\
\end{array}$ \\
\hline Average & & & & $-\ldots \ldots$ & 1,910 & 3,260 & 5,500 & 6,630 & 7,850 & 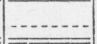 & 260 & 390 & 500 & 490 & 530 \\
\hline $\begin{array}{l}\text { Portland-pozzolan } \\
\quad \text { Do }\end{array}$ & \multirow[t]{2}{*}{$\begin{array}{l}\mathrm{P}-1 \\
\mathrm{P}-2\end{array}$} & \multirow[t]{2}{*}{$\begin{array}{l}2.05 \\
1.88\end{array}$} & \multirow[t]{2}{*}{$\begin{array}{l}50 \\
54\end{array}$} & \begin{tabular}{ll}
$-\ldots+\cdots$ \\
\hdashline \\
\hdashline
\end{tabular} & $\begin{array}{l}1,390 \\
1,760 \\
\end{array}$ & $\begin{array}{l}2,520 \\
2,880 \\
\end{array}$ & $\begin{array}{l}5,610 \\
4,340 \\
\end{array}$ & $\begin{array}{l}6,460 \\
4,960 \\
\end{array}$ & $\begin{array}{l}6,780 \\
5,510 \\
\end{array}$ & $-\ldots$ & $\begin{array}{l}210 \\
225 \\
\end{array}$ & $\begin{array}{l}310 \\
315 \\
\end{array}$ & $\begin{array}{l}480 \\
415 \\
\end{array}$ & $\begin{array}{l}470 \\
385 \\
\end{array}$ & $\begin{array}{l}545 \\
435 \\
\end{array}$ \\
\hline Average & & & & $\bar{\ldots}$ & 1,580 & 2,700 & 4,980 & 5,710 & 6,140 & $\begin{array}{ll}\ldots \ldots \\
-\ldots . . . \\
\end{array}$ & 220 & 310 & 445 & 430 & 490 \\
\hline $\begin{array}{l}\text { High-early strength } \\
\text { Do } \\
\text { Do }\end{array}$ & \multirow[t]{2}{*}{$\begin{array}{l}\text { S-1 } \\
\text { S-2 } \\
\text { S-3 }\end{array}$} & \multirow[t]{2}{*}{$\begin{array}{l}2.05 \\
1.96 \\
1.94\end{array}$} & \multirow[t]{2}{*}{$\begin{array}{l}42 \\
47 \\
47\end{array}$} & $\begin{array}{l}1,750 \\
1,790 \\
1,540 \\
\end{array}$ & $\begin{array}{l}3,580 \\
3,600 \\
3,770 \\
\end{array}$ & $\begin{array}{l}4,680 \\
5,070 \\
4,710\end{array}$ & $\begin{array}{l}5,770 \\
6,120 \\
6,300 \\
\end{array}$ & 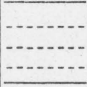 & $\begin{array}{l}7,370 \\
7,150 \\
7,290 \\
\end{array}$ & $\begin{array}{l}210 \\
240 \\
205\end{array}$ & $\begin{array}{l}390 \\
420 \\
410\end{array}$ & $\begin{array}{l}525 \\
520 \\
465\end{array}$ & $\begin{array}{l}530 \\
495 \\
500\end{array}$ & $\begin{array}{l}495 \\
445 \\
490\end{array}$ & $\begin{array}{l}510 \\
415 \\
495\end{array}$ \\
\hline Average & & & & 1,690 & 3,650 & 4,820 & 6,060 & $\begin{array}{ll}\ldots \ldots \\
-\ldots . . . . . \\
\end{array}$ & 7,270 & 220 & 405 & 505 & 510 & 475 & 475 \\
\hline High alumina & $\mathrm{L}$ & 2.05 & 51 & 4,590 & $\mid-\ldots$ & 5,250 & 6,310 & & 4,560 & 375 & 435 & 445 & 350 & 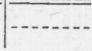 & 305 \\
\hline
\end{tabular}


TABLE 2.-Properties of aerated concretes (mix B: 1:6 by weight)

[Each strength or modulus value is the average for 3 test sperimens]

\begin{tabular}{|c|c|c|c|c|c|c|c|c|c|c|c|c|c|c|c|}
\hline \multirow{2}{*}{ Cement ${ }^{1}$} & \multirow{2}{*}{ Aerating agent } & \multirow{2}{*}{$\begin{array}{l}C / W \text { by } \\
\text { weight }\end{array}$} & \multirow{2}{*}{ Flow } & \multirow{2}{*}{$\begin{array}{l}\text { Weight } \\
\text { of fresh } \\
\text { concrete }\end{array}$} & \multicolumn{5}{|c|}{ Compressive strength at- } & \multicolumn{4}{|c|}{ Tensile strength at- } & \multicolumn{2}{|c|}{$\begin{array}{l}\text { Modulus of elastic- } \\
\text { ity } / 10^{6} \text { at } 28 \text { days }\end{array}$} \\
\hline & & & & & 3 day & 7 day & 28 day & $3 \mathrm{mo}$. & $1 \mathrm{yr}$. & 3 day & 7 day & 28 day & $3 \mathrm{mo}$. & $\underset{\text { sion }}{\text { Compres- }}$ & Tension \\
\hline $\begin{array}{l}\mathrm{A} \\
\mathrm{B} \\
\mathrm{B}\end{array}$ & $\begin{array}{l}\text { None } \\
\text { Sodium lauryl sulfate.- } \\
\text { Rosin }\end{array}$ & $\begin{array}{l}1.66 \\
1.82 \\
1.66\end{array}$ & \begin{tabular}{r|}
$\%_{45}$ \\
46 \\
45
\end{tabular} & \begin{tabular}{r|}
$l b / f t .3$ \\
146.2 \\
137.8 \\
141.2 \\
\end{tabular} & $\begin{array}{ll}b / \text { in. }^{2} \\
1,150 \\
\end{array}$ & $\begin{array}{r}l \text { 's/in. } \\
2,580 \\
2,050 \\
1,970 \\
\end{array}$ & $\begin{array}{r}\text { lb/in.2 } \\
4,650 \\
3,720 \\
3,830 \\
\end{array}$ & $\begin{array}{l}l b / \text { in. }^{2} \\
3,900 \\
\end{array}$ & $\begin{array}{ll}b / \text { in. }^{2} \\
4,540 \\
\end{array}$ & $\begin{array}{r}\text { lb/in. } .^{2} \\
220 \\
190 \\
\end{array}$ & $\begin{array}{r}l b / \text { in. } .^{2} \\
305 \\
290 \\
270 \\
\end{array}$ & $\begin{array}{r}l t / \text { in } .^{2} \\
385 \\
345 \\
360 \\
\end{array}$ & $\begin{array}{r}\text { lb/in. } .^{2} \\
380 \\
355 \\
\end{array}$ & $\begin{array}{r}l b / \text { in. }^{2} \\
4.1 \\
3.6 \\
3.6\end{array}$ & $\begin{aligned} & \\
& \text { lb/in. } .^{2} \\
& 4.0 \\
& 3.3 \\
& 3.5 \\
&\end{aligned}$ \\
\hline $\begin{array}{l}\mathrm{D} \\
\mathrm{E} \\
\mathrm{E} \\
\mathrm{G}\end{array}$ & $\begin{array}{l}\text { Tallow } \\
\text { do }\end{array}$ & $\begin{array}{l}1.74 \\
1.66 \\
1.66 \\
1.68\end{array}$ & $\begin{array}{l}45 \\
43 \\
46 \\
46\end{array}$ & $\begin{array}{l}139.9 \\
141.6 \\
139.6 \\
143.2\end{array}$ & $\begin{array}{l}1,230 \\
1,470 \\
1,210 \\
1,440\end{array}$ & $\begin{array}{l}2,190 \\
2,340 \\
2,180 \\
2,500\end{array}$ & $\begin{array}{l}3,060 \\
4,210 \\
3,430 \\
4,070\end{array}$ & $\begin{array}{l}4,200 \\
4,130 \\
3,790 \\
4,860\end{array}$ & $\begin{array}{l}4,640 \\
4,520 \\
3.940 \\
5,310\end{array}$ & $\begin{array}{l}220 \\
210 \\
185 \\
210\end{array}$ & $\begin{array}{l}275 \\
330 \\
270 \\
300\end{array}$ & $\begin{array}{l}330 \\
325 \\
355 \\
390 \\
\end{array}$ & $\begin{array}{l}385 \\
310 \\
325 \\
415\end{array}$ & $\begin{array}{l}3.6 \\
3.7 \\
3.4 \\
3.7\end{array}$ & $\begin{array}{l}3.5 \\
\text { 3. } 6 \\
3.3 \\
3.6\end{array}$ \\
\hline \multicolumn{2}{|c|}{ A verage } & & ... & 141.1 & 1,340 & 2,400 & 3,690 & 4,250 & 4,600 & 210 & 290 & 350 & 360 & 3.6 & 3. 5 \\
\hline $\begin{array}{l}\mathrm{H} \\
\mathrm{H} \\
\mathrm{I} \\
\mathrm{J}\end{array}$ & $\begin{array}{l}\text { Vinsol resin } \\
\text { do }\end{array}$ & $\begin{array}{l}1.74 \\
1.70 \\
1.77\end{array}$ & $\begin{array}{l}46 \\
45 \\
47\end{array}$ & $\begin{array}{l}142.3 \\
143.6 \\
141.2\end{array}$ & $\begin{array}{l}1,710 \\
1,920 \\
1,350\end{array}$ & $\begin{array}{l}2,710 \\
2,960 \\
2,510\end{array}$ & $\begin{array}{l}4,310 \\
4,380 \\
3,960\end{array}$ & $\begin{array}{l}4,780 \\
5,050 \\
4,630\end{array}$ & $\begin{array}{l}5,340 \\
5,520 \\
5,560\end{array}$ & $\begin{array}{l}255 \\
250 \\
190\end{array}$ & $\begin{array}{l}320 \\
320 \\
250\end{array}$ & $\begin{array}{l}390 \\
350 \\
315\end{array}$ & $\begin{array}{l}405 \\
390 \\
410\end{array}$ & $\begin{array}{l}3.9 \\
3.9 \\
3.7\end{array}$ & $\begin{array}{l}\text { 3. } 6 \\
\text { 3. } \\
\text { 3. } \\
\end{array}$ \\
\hline \multicolumn{2}{|c|}{ Average... } & & ... & 142.4 & 1,660 & 2,730 & 4,160 & 4,820 & 5,470 & 230 & 295 & 350 & 400 & 3.8 & 3.7 \\
\hline
\end{tabular}

1 These cements were of different lots or brands from those listed in table 1 .

2 Normal cement A, with 0.05 percent of a soluble aerating agent added in the laboratory. 


\section{Journal of Research of the National Bureau of Standards}

\section{(b) VARIATION IN STRENGTH FOR CEMENTS WITHIN A TYPE}

There was sometimes considerable variation in strength for different cements within a type, as, for instance, for the two portland-pozzolan cements, table 1. However, for both the normal and the high-earlystrength portland cements, the variation of either compressive or tensile strength from the average for the type was, at any age, less than 16 percent. Somewhat greater variation is found among the moderate-heat cements. The aerated concretes, table 2, also show considerable variation, as is to be expected from the effect of the various aerating agents on the weight per cubic foot of concrete. This varied from 138 to $144 \mathrm{lb}$ compared with $146 \mathrm{lb}$ for the unaerated concrete made with cement $\mathrm{A}$.

\section{(c) VARIATION IN STRENGTH WITH TYPE OF CEMENT}

In figure 2 the average strengths for each type of cement are plotted against age. For both mixes the strengths for the high-early-strength cements, both in tension and compression, for ages up to and including 28 days are higher than for all others, except for the 1:4.5 mix with the high-alumina cement.
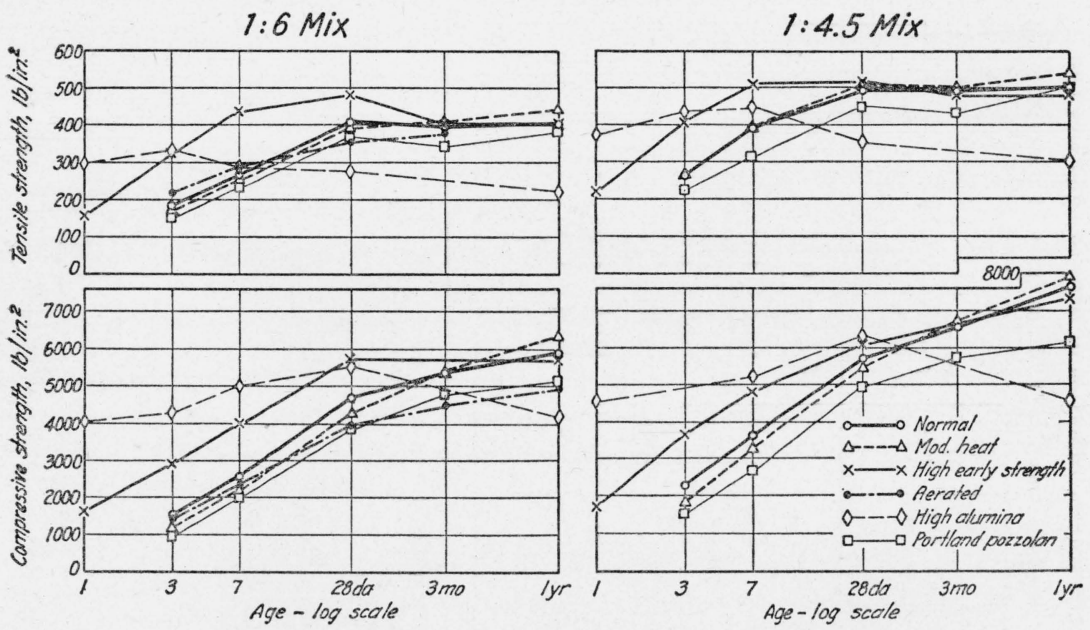

FIGURE 2,-Age-strength relations for concretes made with various types of cement. Strength values are averages for each type of cement.

In figure 2 it may be seen that to attain an average compressive strength of $4,600 \mathrm{lb} / \mathrm{in} .{ }^{2}$ requires about 28 days for the normal cements, 1:6 mix, and about 12 days for the high-early-strength cements in the same mix. Similarly, for an average tensile strength of $400 \mathrm{lb} /$ in. $^{2}$, attained by the normal cements in 28 days, only about 6 days are required by the high-early-strength cements. A similar relation is seen for the 1:4.5 mix. Thus it appears that the use of a high-earlystrength cement is of greater advantage in securing high early tensile than high early compressive strength.

For both the 1:6 and 1:4.5 mixes, the moderate-heat cements gave strengths up to about 28 days somewhat lower than those for the normal cements. After 3 months there is a tendency for the moderate- 
heat cements to give slightly higher strengths, both in tension and compression, than the normal cements.

The aerated concretes, represented only by the 1:6 mix, show a wider range of strengths than the other types, as is to be expected both from the variety of aerating agents used and the number of cements tested. Although the tensile strengths at the early ages are on the average slightly higher than those for the normal cements, at the later ages there is a tendency for both tensile and compressive strengths to fall below those for the normal cements.

The two portland-pozzolan cements differed widely from each other, table 1. For one of these, the strengths up to 7 days were within the range of those for the moderate-heat portland cements; however, after 7 days the strengths for this cement were appreciably lower than those given by the moderate-heat cements. The other portland-pozzolan cement gave relatively low strengths only up to 3 or 7 days, but thereafter the strengths were within the range of those for the normal cements.

The high-alumina cement is unique in showing very high early strength (at 3 days or less). However, compressive strengths began retrogressing some time after 28 days and tensile strengths some time after 3 days for the 1:6 mix and 7 days for the $1: 4.5 \mathrm{mix}$, so that at 1 year the strengths for this cement, both in tension and compression were no higher than at 1 day, and were less than for any other type of cement.

\section{(d) EFFECT OF AGE OF CONCRETE ON STRENGTH}

While the concretes made with two of the three high-early-strength cements (table 1) showed slight retrogression in compressive strength for the 1:6 mix after 28 days, those made with all the other types of cement except the high-alumina showed continued increase in compressive strength, for both mixes, up to a year. Tensile strengths, however, usually reached a maximum value at some time between 7 days and 3 months, followed by retrogression (table 1). The maximum tensile strengths obtained for the 1:6 mix with the highearly-strength cements were higher than those for the same mix with the other cements (fig. 2). However, the high-early-strength cements showed relatively large retrogressions, and, at 3 months and thereafter, gave about the same tensile strengths as the normal cements. For the 1:4.5 mix, two of the three high-early-strength cements gave maximum values at 7 days, but these were no higher than those attained by other cements at later ages. One of the three cements (S-2, table 2) of this type showed large retrogression for this mix.

The average strengths for the moderate-heat cements at 1 year were the highest for all cements tested. For the normal and portlandpozzolan cements, any retrogression between 28 days and 3 months was usually followed by some recovery, so that, at 1 year, the tensile strengths are never more than 10 percent lower than at 28 days, and are often higher.

The retrogressions in tensile strengths of concretes after certain ages may be due to retrogression in mortar tensile strengths, since such retrogression occurs with mortar briquets. The retrogressions may also be due in part to decrease in bond strength between gravel and mortar. Considerable gravel fracture was noted at 28 days, $538330-43-5$ 


\section{Journal of Research of the National Bureau of Standards}

and there was some tendency for the percentage of gravel fractured to decrease with age thereafter.

(e) EFFECT ON STRENGTH OF CEMENT CONTENT OF CONCRETE

In general, the concrete with the higher cement content (1:4.5 mix) gave higher strength, for all cements, than the 1:6 mix, at correspond-

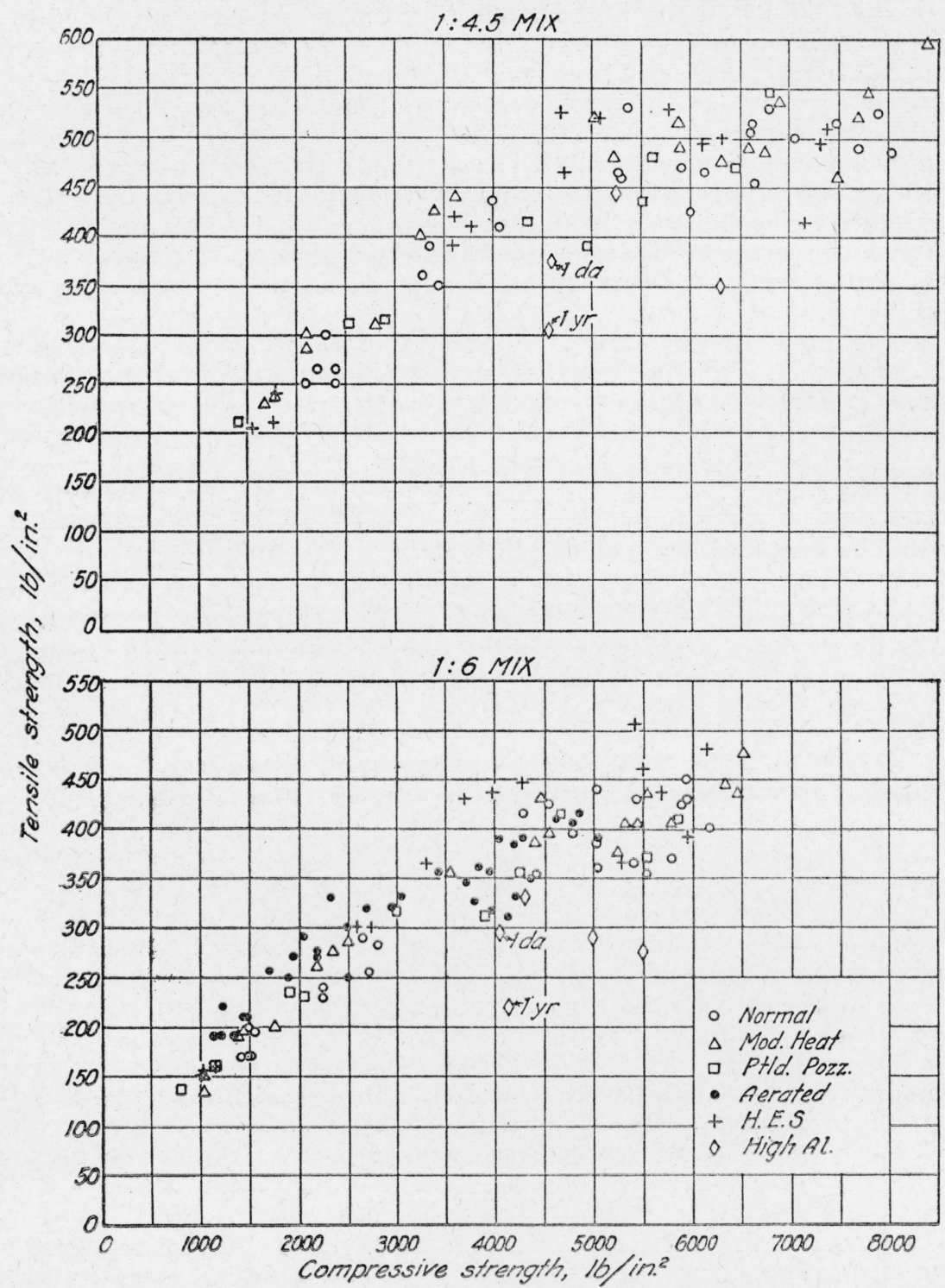

FIGURE 3.-Relations between compressive and tensile strengths.

ing ages. However, there are some noteworthy differences between the rates of increase of tensile and of compressive strength. For instance, comparing the 1:4.5 mix with the 1:6 mix for the normal 
cements, we see (fig. 2 ) that to attain an average compressive strength of $4,600 \mathrm{lb} / \mathrm{in}^{2}$ requires 28 days for the 1:6 mix and about 14 days for the $1: 4.5 \mathrm{mix}$. Similarly, the average tensile strength for the $1: 6$ mix is about $400 \mathrm{lb} /$ in. $^{2}$ at 28 days, and this is attained by the richer concrete in only 8 days. A similar relation for the two mixes with the high-early-strength cements can also be seen. Thus the use of the richer mix is of greater advantage in securing high early tensile than high early compressive strength.

\section{(f) RELATIONS BETWEEN COMPRESSIVE AND TENSILE STRENGTHS}

In figure 3 the tensile strengths have been plotted against the corresponding compressive strengths for each of the two mixes, for all ages. It is seen that there is a fair relation between compressive and tensile strengths.

For equal compressive strengths, tensile strengths tend to be somewhat higher for the $1: 4.5 \mathrm{mix}$ than for the $1: 6$. There is also a tendency for the aerated concretes to have higher tensile strengths for equal compressive strengths up to about $2,500 \mathrm{lb} /$ in. $^{2}$ than the other 1:6 concretes. The high-alumina cement shows appreciably lower tensile strength, for equal compressive strengths, than the other cements.

\section{(g) STRESS-STRAIN RELATIONS}

The stress-strain curves for the 1:6 concretes made with cements containing aerating agents and for cement A the normal cement used for the tests with a soluble aerating agent), are shown in figures 4 and 5 . The cements are arranged in the order of compressive strengths.

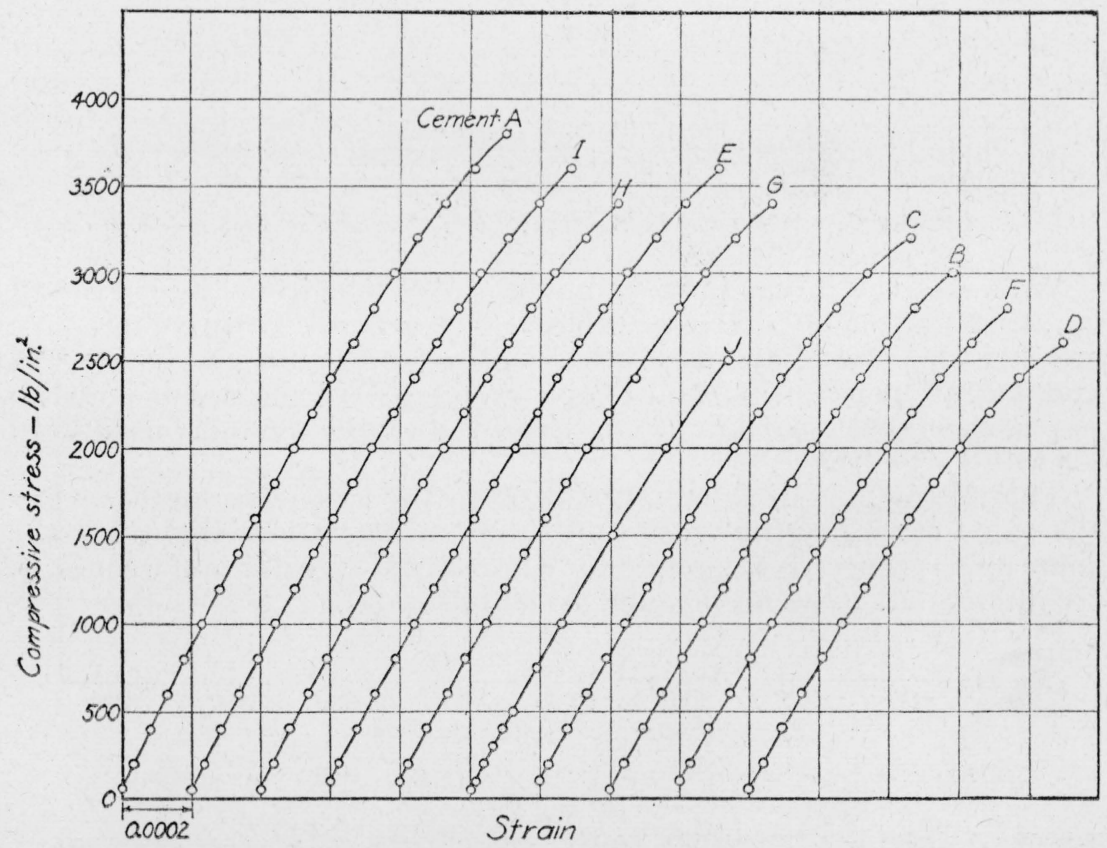

Frgure 4.-Stress-strain relations for 1:6 concretes in compression. 
For the compressive specimens the difference in strain values for opposite sides of the same specimen was usually less than $60 \times 10^{-6}$ for stresses up to three-fourths of the breaking stress; this corresponds to a stress difference of $250 \mathrm{lb} / \mathrm{in} .^{2}$ or less. For the tensile specimens the corresponding difference in strain values was usually less than $10 \times 10^{-6}$, equivalent to a stress difference of $40 \mathrm{lb} / \mathrm{in} .{ }^{2}$ or less. The deviation of the strain for one specimen from the average for a set of three was only about $20 \times 10^{-6}$ for the compressive tests, and about $3 \times 10^{-6}$ for the tensile tests.

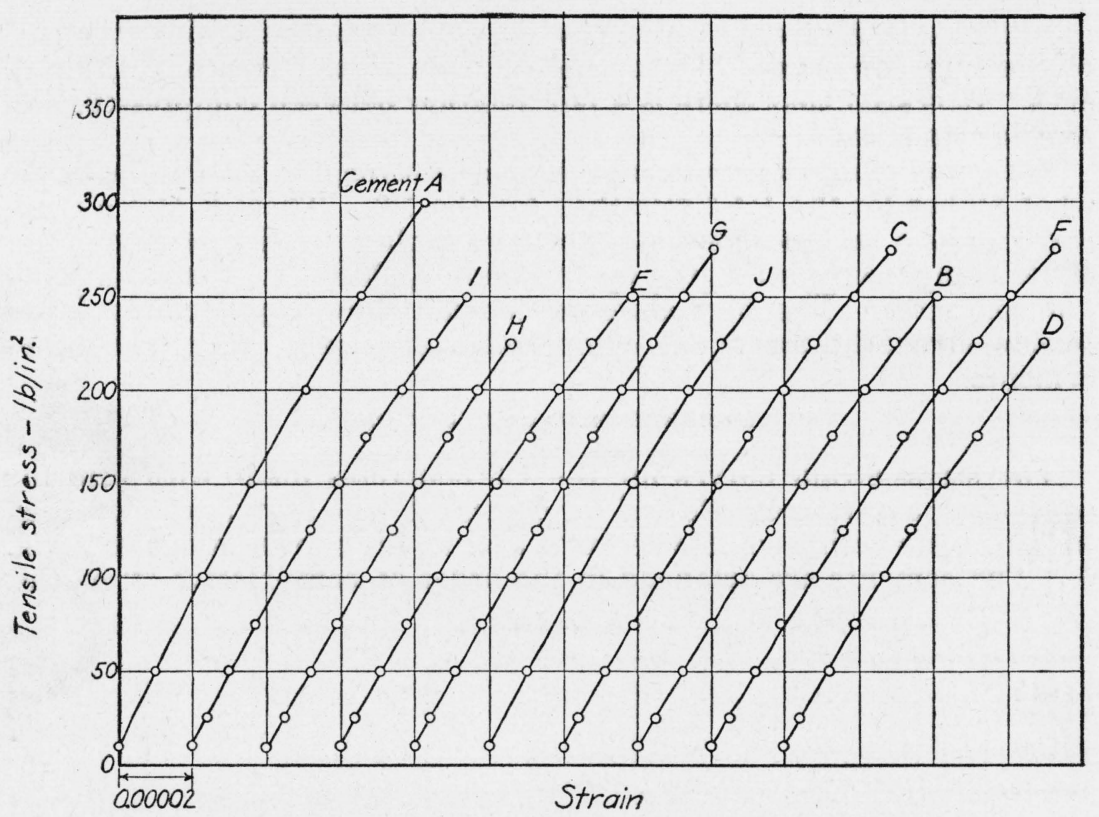

FiguRE 5.-Stress-strain relations for 1:6 concretes in tension.

Values of the initial tangent moduli, obtained from the curves of figures 4 and 5 , are given in table 2 . In general, both compressive and tensile moduli increase with increasing compressive strengths and weights per cubic foot of the concretes. The compressive moduli varied from 3.4 to $4.1 \times 10^{6} \mathrm{lb} / \mathrm{in} .{ }^{2}$, and the tensile moduli varied from 3.3 to $4.0 \times 10^{6} \mathrm{lb} / \mathrm{in}^{2}$.

The tensile moduli show no relation to the tensile strengths. For the same concrete, the tensile modulus appears to be slightly lower than the compressive modulus. Similar but greater differences in secant moduli have been noted by Johnson [7]. 


\section{EFFECT OF SAND-GRAVEL RATIO AND OF VARIOUS COARSE AGGREGATES ON TENSILE STRENGTH}

(a) SAND-GRAVEL RATIO

The results of the tests with Potomac River aggregate in which the sand-gravel ratio was varied are given in table 3 . It is seen that for all three cements, $\mathrm{K}, \mathrm{L}$, and $\mathrm{M}$, the 1:2.9:3.1 mix gave the highest tensile strengths, both at 3 and 28 days (except in one instance where the strength is within $5 \mathrm{lb}$ of the maximum) despite the fact that the water requirement was higher than for the 1:2.4:3.6 mix. The 1:1.9:4.1 mix, which was deficient in sand, gave low tensile strengths despite the high $\mathrm{C} / \mathrm{W}$ ratios. The $1: 3.4: 2.6 \mathrm{mix}$, which was definitely oversanded, had both a high water requirement and low tensile strengths.

TABLE 3.-Tensile strength of concretes with various sand-gravel ratios

[Each strength value is the average for 3 test specimens]

\begin{tabular}{|c|c|c|c|c|c|}
\hline \multirow{2}{*}{ Mix (proportion by weight) ${ }^{1}$} & \multirow{2}{*}{$\underset{\text { wt }}{C / W}$ by } & \multirow{2}{*}{$\begin{array}{l}\text { Water } \\
\text { gal/bag }\end{array}$} & \multirow{2}{*}{$\begin{array}{c}\text { Flow } \\
\%\end{array}$} & \multicolumn{2}{|c|}{ Tensile strength at- } \\
\hline & & & & $\begin{array}{l}3 \text { days } \\
\text { lb/in. }{ }^{2}\end{array}$ & $\begin{array}{l}28 \text { days } \\
\mathrm{lb} / \mathrm{in}^{2}\end{array}$ \\
\hline \multicolumn{6}{|c|}{ CEMENT K 2} \\
\hline $\begin{array}{l}1: 1.9: 4.1 \\
1: 2.4: 3.6 \\
1: 2.9: 3.1 \\
1: 3.4: 2.6 \ldots-2\end{array}$ & $\begin{array}{l}1.73 \\
1.66 \\
1.53 \\
1.42\end{array}$ & $\begin{array}{l}6.5 \\
6.8 \\
7.4 \\
7.9\end{array}$ & $\begin{array}{l}48 \\
49 \\
52 \\
49\end{array}$ & $\begin{array}{l}145 \\
180 \\
190 \\
160\end{array}$ & $\begin{array}{l}325 \\
380 \\
430 \\
380\end{array}$ \\
\hline
\end{tabular}

CEMENT L 2

\begin{tabular}{l|l|l|l|l|l}
\hline $1: 1.9: 4.1$ & 1.73 & 6.5 & 49 & 180 \\
$1: 2.4: 3.6$ & 1.66 & 6.8 & 44 & 420 \\
$1: 2.9: 3.1$ & 1.53 & 7.4 & 43 & 240 \\
$1: 3.4: 2.6$ & 1.42 & 7.9 & 55 & 460 \\
\end{tabular}

CEMENT M 2

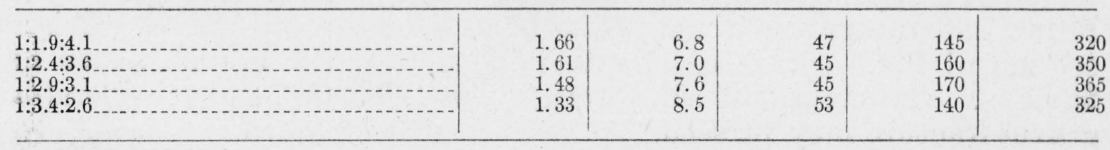

1 Made of cement, sand, and $3 / 4$-in. gravel.

2 Normal portland cements of different brands or lots from those listed in tables 1 and 2.

(b) COARSE AGGREGATES

In table 4 are given the results of the tests with various coarse aggregates.

For both mixes, Potomac gravel gave the highest strengths of any aggregate at 3 and 7 days, even when the lower $C / W$ ratio was used. At 28 days the Potomac gravel gave higher strength than the other aggregates in the 1:6 mix, but the New York limestone gave the highest value for the 1:4.5 mix. Both the trap-rock and granite gave fairly low values for the $1: 4.5 \mathrm{mix}$ at 28 days, whereas the slag, which had about the same bulk specific gravity as Potomac gravel, gave a rather high value. 
TABLE 4.-Tensile strengths of concretes with various coarse aggregates

[Each strength value is the average for 3 test specimens.]

\begin{tabular}{|c|c|c|c|c|c|c|c|}
\hline \multirow{2}{*}{ Coarse aggregate } & \multirow{2}{*}{$\begin{array}{l}\text { Proportions }{ }^{1} \\
\text { by dry wt }\end{array}$} & \multirow{2}{*}{$\begin{array}{l}\mathrm{C} / \mathrm{W} \\
\text { by wt }\end{array}$} & \multirow{2}{*}{ Water } & \multirow{2}{*}{ Flow } & \multicolumn{3}{|c|}{ Tensile strength ${ }^{2}$ at- } \\
\hline & & & & & 3 days & 7 days & 28 days \\
\hline $\begin{array}{l}\text { Potomac gravel } \\
\text { Do } \\
\text { N. Y. limestone } \\
\text { Ohio slag } \\
\text { Potomac gravel } \\
\text { N. Y. granite } \\
\text { N. Y. limestone } \\
\text { Pa. "trap-rock"- } \\
\text { Ohio slag }\end{array}$ & $\begin{array}{c}1: 2.4: 3.6 \\
1: 2.4: 3.6 \\
1: 2.4: 4.0 \\
1: 2.4: 3.6 \\
1: 1.75: 2.75 \\
1: 1.75: 2.80 \\
1: 1.75: 3.00 \\
1: 1.75: 3.00 \\
1: 1.75: 2.75\end{array}$ & $\begin{array}{l}1.66 \\
1.48 \\
1.48 \\
1.48 \\
2.05 \\
1.88 \\
1.88 \\
1.88 \\
1.88\end{array}$ & $\begin{array}{r}\text { gal } / \text { bag } \\
6.8 \\
7.6 \\
7.6 \\
7.6 \\
5.5 \\
6.0 \\
6.0 \\
6.0 \\
6.0\end{array}$ & $\begin{array}{r}\text { percent } \\
41 \\
78 \\
43 \\
40 \\
40 \\
35 \\
40 \\
39 \\
35\end{array}$ & $\begin{array}{l}\text { lb/in. } \\
315 \\
205 \\
205 \\
230 \\
230\end{array}$ & $\begin{array}{r}\text { lb/in.2 } \\
280 \\
240 \\
175 \\
195 \\
-2 \\
-2 \\
-2\end{array}$ & $\begin{array}{r}\text { lb/in. }{ }^{2} \\
445 \\
385 \\
315 \\
380 \\
445 \\
405 \\
525 \\
380 \\
460\end{array}$ \\
\hline
\end{tabular}

1 The amounts of coarse aggregates were varied in proportion to their bulk specific gravities.

2 The normal cement used for these tests was different from those of the previous tables.

For the 1:4.5 mix, the 28-day tensile strengths vary over a range of almost 1.4 to 1 , depending on the coarse aggregate. Kellerman [11] has noted a similar variation, as well as the fact that relative tensile strengths for different aggregates depend on the richness of the mix. The New York limestone, for instance, gave the highest 28-day tensile strength in the rich mix, but the lowest in the leaner mix. A possible explanation is, that with some aggregates, such as Potomac gravel, the bond strength between mortar and coarse aggregate may be relatively high; this would tend to give relatively high strength at the early ages, when little of the coarse aggregate is broken. With the New York limestone, on the other hand, bond strength may be relatively low with the leaner mix, resulting in low strength at the early ages; but this aggregate may itself have higher strength and, when used in a richer mix tending to cause failure of the aggregate, the concrete strength may be rather high.

Some of the fractured sections are shown in figures 6 and 7 . Figure $6, A$, is a section of $1: 6$ aerated concrete made with Potomac gravel, tested at 3 days. This concrete had an average tensile strength of 220 $\mathrm{lb} / \mathrm{in}^{2}$, and the failure is mainly in bond of mortar to gravel. $B$ is a section of 1:4.5 concrete, also made with Potomac gravel, tested at 28 days. The average tensile strength was $480 \mathrm{lb} / \mathrm{in}^{2}$, and considerable gravel fracture may be seen.

In figure $7, A$ is a section of 1:6.4 concrete made with New York limestone and tested at 28 days. The average tensile strength was $315 \mathrm{lb} / \mathrm{in}^{2}$, and very little of the stone was fractured. $B$ is a section of $1: 4.75$ concrete made with the same aggregate and tested at 28 days. Here the tensile strength was $525 \mathrm{lb} / \mathrm{in}^{2}$, and some aggregate fracture occurred.

\section{SUMMARY}

1. An improved tensile-test method for concrete has been developed. Cylindrical specimens, 4 by 16 in. are used, and loads are applied through threaded rods embedded in a rich mortar at the ends of the specimens. The method gave fairly uniform results because of the greater uniformity of stress distribution over the section of failure than is obtained with grips, or in tapered specimens, etc. 

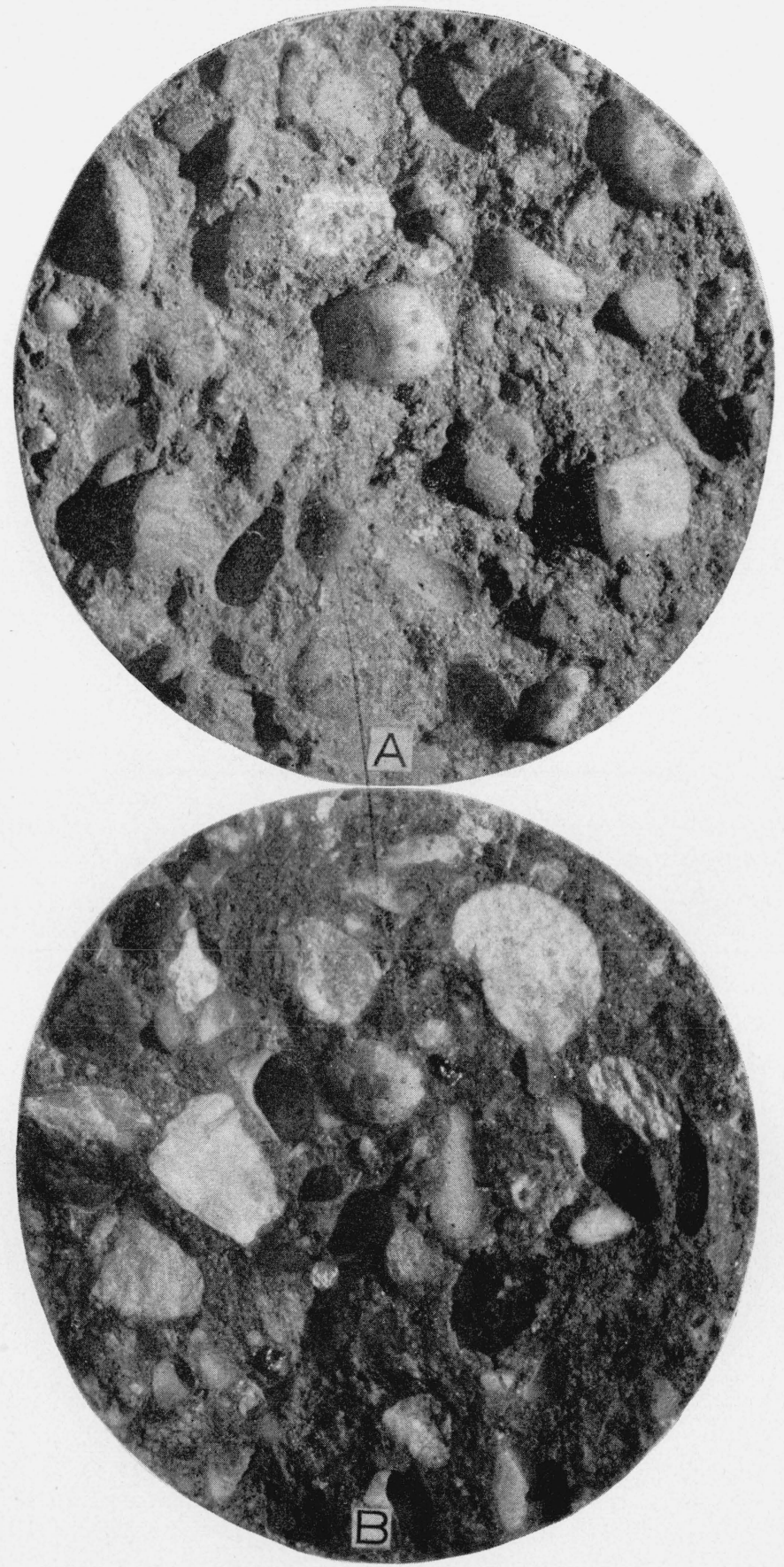

Figure 6.-Fractured sections of Potomac River gravel concrete. 


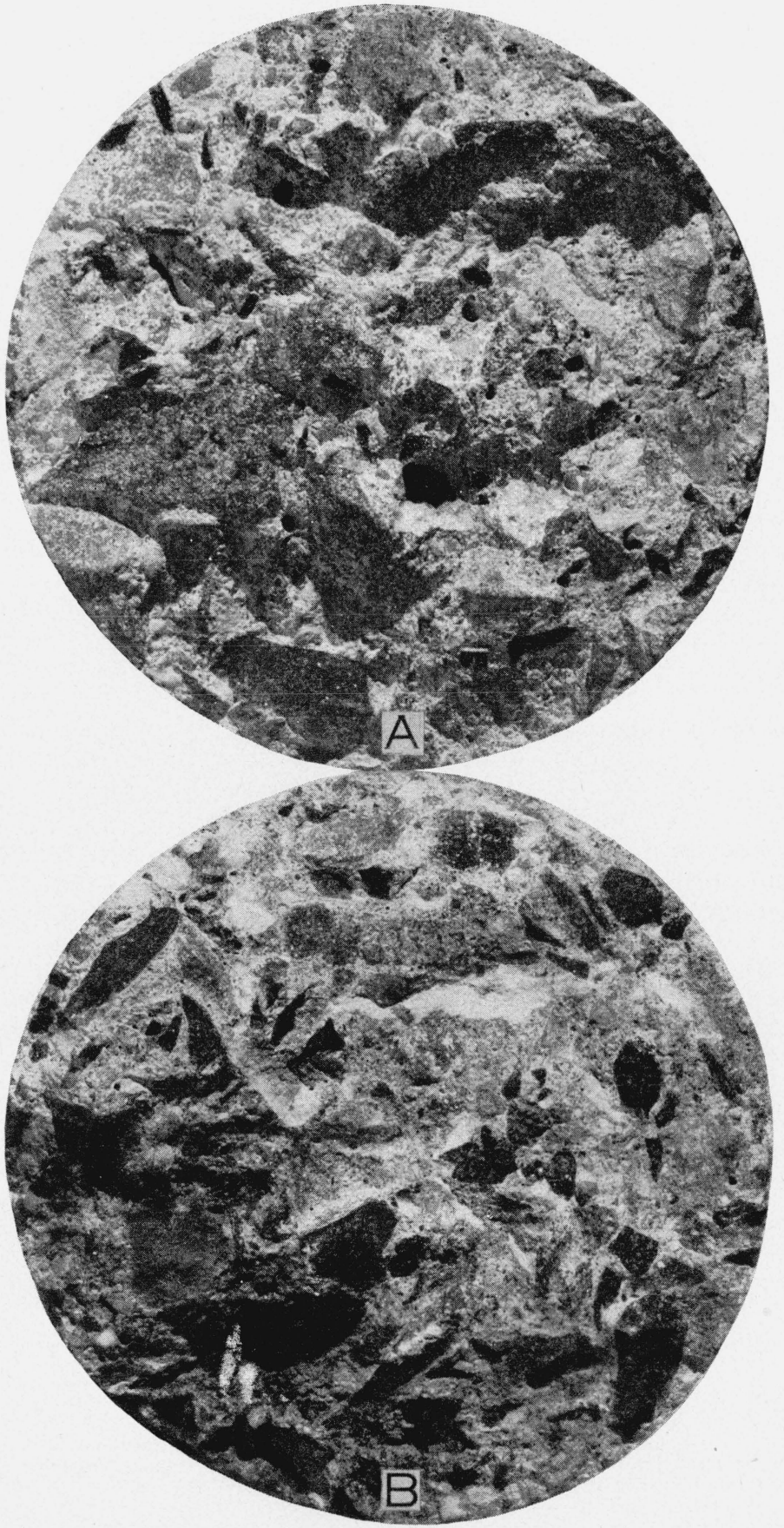

Figure 7.-Fractured sections of New York limestone concrete. 
2. Compressive and tensile strengths and some stress-strain relations were determined for concretes made with a number of cements of various types, including five normal, four moderate-heat, and three high-early-strength portland cements, two portland-pozzolans, one high-alumina cement, and nine portland cements with aerating agents. The effects of various sand-gravel ratios and of various coarse aggregates on tensile strength were also studied.

3. The high-early-strength cements gave higher strengths than the normal cements, both in tension and compression, up to 28 days. The moderate-heat cements gave but slightly lower strengths than the normal cements up to 28 days; thereafter there was some tendency for the moderate-heat cements to give higher compressive and tensile strengths than the normal cements. The cements with aerating agents gave somewhat higher tensile strengths than the normal cements up to 7 days, but thereafter both compressive and tensile strengths were lower than those for the normal cements. Of the two portland-pozzolans tested, one gave rather low early strength but came within the range for the normal cements at later ages; the other gave early strengths comparable to a moderate-heat portland but gave relatively low strengths at later ages. The high-alumina cement gave very high strengths at 1 day but showed comparatively little gain at later ages.

4. Tensile strengths usually attained maximum values between 7 days and 3 months, with retrogression in many cases. However, at 1 year, tensile strengths were generally within \pm 10 percent of the 28-day strengths, except for the high-alumina cement, which gave appreciably lower values. The fracture sections of the tensile specimens often showed considerable aggregate breakage at 28 days, and somewhat less at later ages.

5. The 1:4.5 mix generally gave higher strengths for all cements than the 1:6 mix at the same age. The use of either a richer mix with the normal cements, or of a high-early-strength cement, was of greater advantage in securing high early tensile strength than high early compressive strength.

6. Tensile and compressive strengths show a fair relation, but the 1:4.5 mix gave somewhat higher tensile strengths for equal compressive strengths than the 1:6 mix. For compressive strengths up to about $2,500 \mathrm{lb} /$ in. $^{2}$, the $1: 6$ aerated concretes gave somewhat higher tensile strengths for equal compressive strengths than the 1:6 mix with normal cements. The high-alumina cement gave appreciably lower tensile strengths for equal compressive strengths than the other cements.

7. For the nine aerated 1:6 concretes tested with Potomac River sand and gravel, the weights per cubic foot ranged from 138 to $144 \mathrm{lb}$. compared with 146 for a corresponding normal cement concrete. Initial tangent moduli of elasticity for the aerated concretes ranged from 3.4 to $3.9 \times 10^{6} \mathrm{lb} / \mathrm{in}^{2}$ in compression, and from 3.3 to $3.8 \times 10^{6}$ $\mathrm{lb} /$ in. $^{2}$ in tension, compared with 4.1 and 4.0 , respectively, for a normal cement concrete. Both the tensile and compressive moduli increased with compressive strengths, which ranged from 3,100 to $4,600 \mathrm{lb} /$ in. $^{2}$ at 28 days.

8. Tensile strengths of concrete varied considerably with the sandgravel ratio or with the type of coarse aggregate used. A range of 1.4 to 1 in tensile strength at 28 days was noted for different coarse 
aggregates, such as slag, crushed stones, and gravel. A crushed limestone gave relatively low tensile strength in a 1:6.4 mix, and rather high strength in a 1:4.8 mix.

Acknowledgments are due the various cement manufacturers, the National Crushed Stone Association, and the National Slag Association for the materials contributed for use in this investigation.

\section{REFERENCES}

[1] Standard method of test for compressive strength of concrete, Am. Soc. Testing Materials Standards, 1939 , pt. 2, Nonmetallic materials, constructional, p. 328. ASTM Designation C39-39.

[2] A. N. Talbot, Tests of Reinforced Concrete Beams. Univ. Ill. Eng. Exp. Sta. Bul. No. 1 (September 1, 1904).

[3] M. O. Withey, Tests of plain and reinforced concrete, Series of 1907, Univ. Wis. Eng. Series, 4, No. 2, 67-136, Bul. 197.

[4] A. N. Johnson, Concrete in tension. Proc. Am. Soc. Testing Materials 26, 441 , pt. 2 (1926).

[5] H. F. Gonnerman and E. C. Shuman, Compression, flexure, and tension tests of plain concrete, Proc. Am. Soc. Testing Materials 28, 527, pt. 2 (1928).

[6] R. E. Davis, Discussion [5, p. 556].

[7] J. W. Johnson, Relationship between strength and elasticity of concrete in tension and compression. Iowa State Coll. Eng. Exp. Sta. Bul. 90, 26, No. 72 (May 9, 1928).

[8] A. Guttman, Zur Frage der Zugfestigkeit unbewehrten Beions, Zement 24, No. 35 (Aug. 29, 1935).

[9] R. Dutron, Les Matières intertes et les propriétés mécaniques des bétons, Rev. matériaux construction trav. publies, 26 (1930), No. 253.

[10] L. B. Tuckerman, Optical strain-gages and extensometers, Proc. Am. Soc. Testing Materials, 23, pt. 2, 602 (1923).

[11] W. F. Kellerman, Effect of type and gradation of coarse aggregate upon the flexural and tensile strength of plain concrete, Public Roads 10, No. 5 (June 1929).

Washington, March 24, 1943. 
NATIONAL BUREAU OF STANDARDS, WASHINGTON, D. C.

Send me the Mathematical Tables marked X below. I enclose remittance ${ }^{1}$ to cover the cost.

\begin{tabular}{|c|c|c|c|c|}
\hline Mark X & Title of publication & $\begin{array}{l}\text { United States and } \\
\text { its possessions, } \\
\text { and countries ex- } \\
\text { tending franking } \\
\text { privilege }\end{array}$ & $\begin{array}{l}\text { Other } \\
\text { countries }\end{array}$ & Amount enclosed \\
\hline (1) & 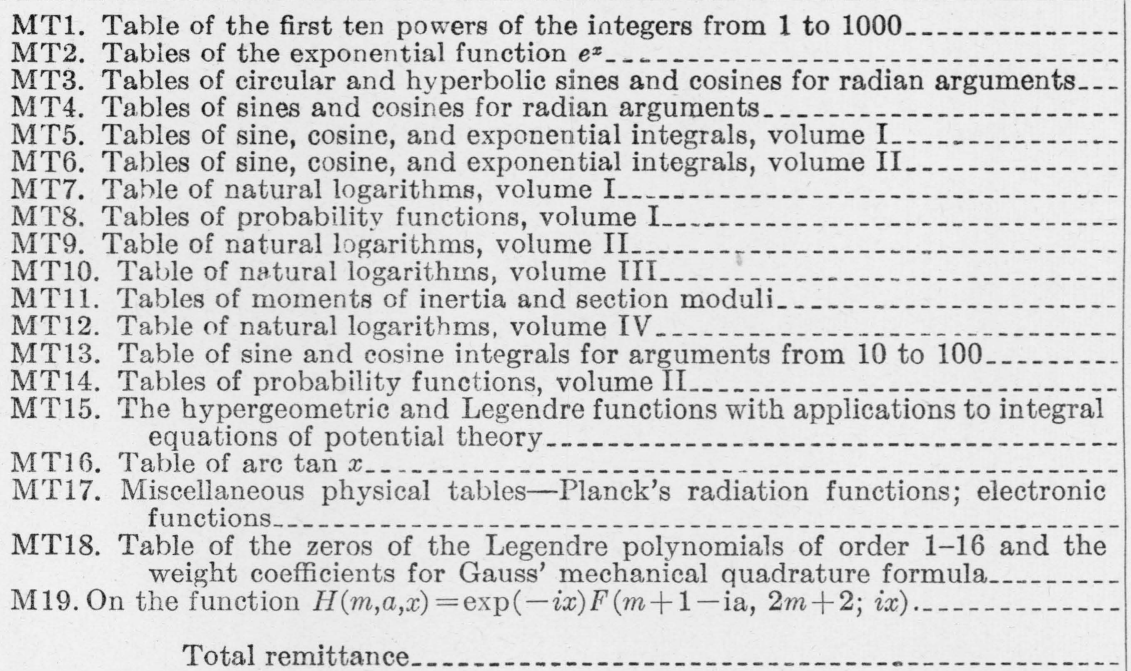 & $\begin{array}{r}\$ 0.50 \\
2.00 \\
2.00 \\
2.00 \\
2.00 \\
2.00 \\
2.00 \\
2.00 \\
2.00 \\
2.00 \\
2.00 \\
2.00 \\
2.00 \\
2.00 \\
2.00 \\
2.00 \\
1.50\end{array}$ & $\begin{array}{r}\$ 0.65 \\
2.50 \\
2.50 \\
2.50 \\
2.50 \\
2.50 \\
2.50 \\
2.50 \\
2.50 \\
2.50 \\
2.50 \\
2.50 \\
2.50 \\
2.50 \\
2.50 \\
2.50 \\
1.75 \\
\\
.30 \\
.30\end{array}$ & $\begin{array}{l} \\
\end{array}$ \\
\hline
\end{tabular}

${ }^{1}$ Remittance should be in form of post-office money order, or check, and made payable to the order of the "National Bureau of Standards" in United States curreney.

\section{Send to}

Number and Street

City and State. 


\section{MATHEMATICAL TABLES}

Attention is invited to a series of publications prepared by the Project for the Computation of Mathematical Tables conducted by the Federal Works Agency, Work Projects Administration for the City of New York, under the sponsorship of the National Bureau of Standards. The tables which have been made avail able through the National Bureau of Standards are listed below.

There is included in this list a publication on the hypergeometric and Legendre functions (MT15), prepared by the Bureau.

MT1. Table of the Frrst Ten:Powers of the Integrrs From 1 to 1000:

(1938) VIII+80 pages; heavy paper cover. 50 cents.

MT2. Tables of the Expongntial Function $e^{x}$.

The ranges and intervals of the argument and the number of decimal places in the entries are given below:

$\begin{array}{rcc}\text { Range of } x & \text { Interval of } x & \text { Decimals given } \\ -2.5000 \text { to } 1.0000 & 0.0001 & 18 \\ 1.0000 \text { to } 2.5000 & .0001 & 15 \\ 2.500 \text { to } 5.000 & .001 & 15 \\ 5.00 \text { to } 10.00 & .01 & 12\end{array}$

(1939) XV+535 pages; bound in buckram, $\$ 2.00$.

MT3. Tables of Circular and Hyperbolic Sines and Cosings por Radian Argument8:

Contains 9 decimal place values of $\sin x, \cos x, \sinh x$ and $\cosh x$ for $x$ (in radians) ranging from 0 to 2 at intervals of 0.0001 .

(1939) XVII+ 405 pages; bound in buckram, $\$ 2.00$.

MT4. Tables or Sines and Cosines for Radian Arguments:

Contains 8 decimal place values of sines and cosines for radian arguments ranging from 0 to 25 at intervals of 0.001 .

(1940) XXIX + 275 pages; bound in buckram, $\$ 2.00$.

MT5. Tables of Sine, Cosine, and Exponential Intrgrals, Volume I:

Values of these functions to 9 places of decimals from 0 to 2 at intervals of 0.0001 .

(1940) XXVI+444 pages; bound in buckram, $\$ 2.00$.

\section{MT6. Tables or Sine, Cosine, and Exponential Intrgrals, Volume II:}

Values of these functions to 9,10 , or 11 significant figures from 0 to 10 at intervals of 0.001 , with auxiliary tables.

(1940) XXXVII + 225 pages; bound in buckram, $\$ 2.00$.

MT7. Table of Natural Logarithms, Volumb I:

Logarithms of the integers from 1 to 50,000 to 16 places of decimals.

(1941) XVIII + 501 pages; bound in buckram, $\$ 2.00$.

MT8. Tables of Probability Functions, Volumb I:

Values of these functions to 15 places of decimals from 0 to 1 at intervals of 0.0001 and from 1 to 5.6 at intervals of 0.001 .

(1941) XXVIII + 302 pages; bound in buckram, $\$ 2.00$.

[Continued on p. 4 of cover] 
MT9. Tablr of Natural Logarithme, Volumb II:

Logarithms of the integers from 50,000 to 100,000 to 16 places of decimals.

(1941) XVIII+501 pages; bound in buckram, $\$ 2.00$.

MT10. Table or Natural Logarithms, Volume III:

Logarithms of the decimal numbers from 0.0001 to 5.0000 , to 16 places of decimals.

(1941) XVIII+501 pages; bound in buckram, $\$ 2.00$.

MT11. Tablas of thr Moments of Inertia and Section Moduli or Ordinary Angles, Chan nels, and Bulb Angles With Certain Plate Combinations:

(1941) XIII + 197 pages; bound in green cloth, $\$ 2.00$.

MT12. Table of Natural Logarithms, Volumb IV:

Logarithms of the decimal numbers from 5.0000 to 10.0000 , to 16 places of decimals.

(1941) XXII+ 506 pages; bound in buckram, $\$ 2.00$.

MT13. Table of Sing and Cosine Intrgrals for Argumrnts From 10 to 100:

(1942) XXXII+185 pages; bound in buckram, $\$ 2.00$.

MT14. Tables of Probability Functions, Volumb II:

Values of these functions to 15 places of decimals from $0.0^{-10} 1$ at intervals of 0.0001 and from 1 to 7.8 at intervals of 0.001 .

(1942) XXI + 344 pages; bound in buckram, $\$ 2.00$.

MT15. The hypergeometric and Legendre functions with applications to integral equations of potential theory. By Chester Snow, National Bureau of Standards. Reproduced from original handwritten manuscript.

(1942) VII+319 pages; bound in heavy paper cover, $\$ 2.00$.

MT16. TABLB or Arc Tan X:

Table of inverse tangents for positive values of the angle in radians. Second central differences are included for all entries.

$\begin{array}{rrc}\text { Range of } x & \begin{array}{c}\text { Interval between } \\ \text { successive arguments }\end{array} \\ 0 \text { to } & 7 & 0.001 \\ 7 \text { to } & 50 & .01 \\ 50 \text { to } & 300 & .1 \\ 300 \text { to } 2,000 & 1 \\ 2,000 \text { to } 10,000 & 10\end{array}$

(1942) XXV+169 pages; bound in buckram, $\$ 2.00$.

MT17. Miscellaneous Physical Tables:

Planck's radiation functions (Originally published in the Journal of the Optical Society of America, February 1940); and

Electronic functions.

(1941) VII + 58 pages; bound in buckram, $\$ 1.50$.

MT18. Table of the Zeros of the Legendre Polynomials of Order 1-16 and the Weight Coefficients for Gauss' Mechanical Quadrature Formula.

(Reprinted from Bull. Amer. Mathemical Society, October 1942.)

5 pages with cover. 25 cents.

MT19. On the Function $H(m, a, x)=\exp (-i x) F(m+1-i a, 2 m+2 ; i x)$; with table of the confluent hypergeometric function and its first derivative.

(Reprinted from Journal of Mathematics and Physics, December 1942.) 20 pages, with cover. 25 cents.

Payment is required in advance. Make remittance payable to the "National Bureau of Standards," and send with order, using the blank form facing page 3 of the cover.

The prices are for delivery in the United States and its possessions and in countries extending the franking privilege. To other countries the price of MT1 is 65 cents; that of MT2 to MT16, inclusive, is $\$ 2.50$ each; MT17, $\$ 1.75$; MT18 and MT19, 30 cents each; remittance to be made payable in United States currency.

Copies of these publications have been sent to various Government depositories throughout the country, such as public libraries in large cities, and colleges and universities, where they may be consulted.

A mailing list is maintained for those who desire to receive announcements regarding new tables as they become available. 